\title{
Influencia del Color y Estados de Madurez sobre la Textura de Frutas Tropicales (Mango, Papaya y Plátano)
}

\author{
Ramiro Torres*, Everaldo J. Montes, Omar A. Pérez, Ricardo D. Andrade \\ Grupo de Investigación GIPPAL, Facultad de Ingeniería, Departamento de Ingeniería de Alimentos, \\ Universidad de Córdoba, Km 12 Vía Cereté-Ciénaga de Oro, Colombia (rtorres@correo.unicordoba.edu.co) \\ ${ }^{*}$ Autor a quien debe ir dirigida la correspondencia.
}

Recibido Oct. 17, 2014; Aceptado Dic. 23, 2014; Versión final recibida Ene. 25, 2014

\section{Resumen}

En este estudio se determinó la influencia del estado de madurez sobre las propiedades texturales de mango variedades Puerco y Corazón, de papaya variedad Hawaiana y de plátano. Se correlacionaron los parámetros texturales con el color medido en el espacio CIELAB, mediante un Análisis de Perfil de Textura (dureza, gomosidad, adhesividad, elasticidad, cohesividad y masticabilidad), utilizando un texturómetro con plato de compresión de $50 \mathrm{~mm}$ y una velocidad de compresión de $1 \mathrm{~mm} / \mathrm{s}$. La dureza, gomosidad y masticabilidad pueden correlacionarse con una ecuación polinómica de segundo grado en ${ }^{\circ} \mathrm{HUE}$, para el mango (Mangifera indica L) variedad Puerco y Corazón; la dureza y gomosidad para la papaya (Carica papaya L.) variedad Hawaiana y con la dureza para plátano (Mussa Cavendish).

Palabras clave: frutas tropicales, dureza, gomosidad, masticabilidad, textura, CIELAB

\section{Influence of Color and Maturity Stages on the Texture of Tropical Fruits (Mango, Papaya and Plantain)}

\begin{abstract}
The influence of the stage of maturity on the textural properties of Mango varieties Pork and Heart, of papaya of Hawaiian variety and plantain were determined in this study. Texture Profile Analysis (hardness, gumminess, adhesiveness, springiness, cohesiveness and chewiness) was performed with a texturometer with a compression plate of $50 \mathrm{~mm}$ and a compression rate of $1 \mathrm{~mm} / \mathrm{s}$. Hardness, gumminess and chewiness are well correlated with a second degree polynomial in ${ }^{\circ} \mathrm{HUE}$ for mango (Mangifera indica $\mathrm{L}$ ) variety Puerco and Corazón; hardness and gumminess for papaya (Carica papaya L.) variety Hawaiana y with hardness for plantain (Mussa Cavendish).
\end{abstract}




\section{INTRODUCCIÓN}

Aunque muchos estudios sobre reología y textura de productos vegetales han sido realizados a nivel internacional, poco se ha publicado referente a la caracterización reológica de frutas tropicales. Solo ha sido estudiada en forma puntual la fractura y firmeza en frutas tropicales como uchuva (Ciro y Osorio, 2008), lulo (Ospina et al., 2007), plátano y banano (Ciro et al., 2008; Kajuana et al., 1997), tomate de árbol (Ciro et al., 2005), manzana (Álvarez et al., 2002), granadilla (Ciro et al., 2003), y aguacate (Correa et al., 1995). En Colombia existe un escaso conocimiento del comportamiento reológico de la mayoría de los productos agropecuarios, aunque existen técnicas instrumentales como las imitativas, las cuales son más utilizadas en la industria, porque se ha encentrado que están relacionadas con la calidad de la textura (Bourne, 2002), lo cual permiten desarrollar nuevas técnicas de procesamiento, transformación agroindustrial y manejo poscosecha (Ciro et al., 2007; Zuñiga et al., 2007).

A nivel de investigación, e incluso industrial, la determinación de las propiedades reológicas y texturales se hace con equipos especializados y personal entrenado, lo cual ocasiona altos costo de análisis. Sin embargo, estas se pueden relacionar con atributos o propiedades que sean fáciles de medir y que no impliquen análisis destructivos, como por ejemplo el color, que además de desempeñar un papel importante en la evaluación externa de la calidad del producto (Segnini et al., 1999; Abdullah et al., 2001), se puede analizar rápidamente por un sistema de adquisición de imágenes (Mendoza et al., 2006), también conocido como sistema de visión computacional (SVC), utilizado para identificar, clasificar, evaluar y detectar defectos en frutas, verdura, carne, pescado, productos de panadería, y productos preparados, como chips de papas fritas entre otros (Pedreschi et al., 2004).

El objetivo de este trabajo de investigación fue correlacionar el color, determinado por la técnica de visón computacional, y el índice de madurez con las propiedades texturales del mango (Mangifera indica L), papaya (Carica papaya L.), y plátano (Mussa Cavendish); definir los rangos de color para diferentes estados de madurez, teniendo en cuenta la diferencia de color medida en el espacio de color CILEAB con la formulas CIE 1994 y CIE 2000 (Sharma et al., 2005), y determinar cómo influye el estado de madurez sobre las propiedades texturales del mango, papaya y plátano.

\section{MATERIALES Y MÉTODOS}

\section{Materia Prima}

Se utilizaron frutos de mango (Mangifera indica L) en las variedades "Puerco" y "Corazón", papaya Hawaiana (Carica papaya L.) y plátano (Mussa Cavendish), provenientes de Montería, Córdoba (Colombia), con diferentes estados de madurez, los cuales fueron identificados visualmente por el color de la cáscara mediante la escala de maduración, para mango (Peña, 2008), plátano (Dadzie y Orchard, 1997), y papaya (Santamaría et al., 2009).

\section{Analisis del perfil de textura (APT)}

Se realizó un APT al mango sobre la fruta entera, la cual se sometió a doble compresión, mediante el plato de compresión de $50 \mathrm{~mm}$ de diámetro a una velocidad de $1 \mathrm{~mm} / \mathrm{s}$, hasta $20 \%$ respecto a la altura inicial de la muestra para la primera y segunda compresión, con un tiempo de espera entre etapas de compresión de 5 segundos; a la papaya y plátano pelado se le realizó el APT sobre muestras cilíndricas de $2 \mathrm{~cm}$ de diámetro y $3 \mathrm{~cm}$ de altura, las cuales se sometieron a doble compresión, mediante el plato de compresión de $50 \mathrm{~mm}$ de diámetro a una velocidad de $1 \mathrm{~mm} / \mathrm{s}$, hasta el $30 \%$ respecto a la altura inicial de la muestra para la primera y segunda compresión, con un tiempo de espera entre las etapas de compresión de 5 segundos, en un Texturómetros Shimadzu EZ Test ${ }^{\circledR}$ (Shimadzu Corporation, Tokio, Japón), usando el software RheoMeter ${ }^{\circledR}$ Versión 2.04. Se seleccionó la compresión de 20 y 30 \%, porque a mayor deformación las muestras colapsan y se obtiene parámetros no representativos (Huang et al., 2007).

Para el mango se realizó un diseño unifactorial en bloques completamente al azar, en seis niveles de estados de madurez, EM (1, 2, 3, 4, 5 y 6), bloqueando el factor variedad ("Puerco" y "Corazón"), con 3 réplicas (36 unidades experimentales); para la papaya y plátano el experimento fué conducido bajo un diseño completamente al azar, con factores de efectos principales, y de efecto fijos, el factor EM en 7 niveles $(1,2,3,4,5,6$ y 7$)$, y el factor sentido de la carga en dos niveles (transversal y perpendicular a la dirección de la fibra), se realizaron 3 repeticiones (42 unidades experimentales), evaluando las variables de respuestas dureza, gomosidad, adhesividad, elasticidad, cohesividad y masticabilidad.

Se realizó un ANOVA a un nivel de significancia al 5\%, para determinar la influencia del EM y la variedad sobre las propiedades texturales del mango; la influencia del EM y el sentido de la carga sobre los 
parámetros texturales de la papaya y plátano. Con un análisis de regresión se determinaron los modelos que relacionan el color ( $L^{*}, a^{*}, b^{*}, C^{*}$ y ${ }^{\circ H U E}$ ) con las propiedades texturales del mango variedad "Puerco" y "Corazón", papaya variedad Hawaiana y plátano. Finalmente se determinaron los parámetros de los modelos. La validez de los modelos se evaluó utilizando los parámetros estadísticos de coeficiente determinación y el análisis de los residuos del modelo. El ANOVA y la interpretación estadística se realizó con el Software STATISTICA ${ }^{\circledR}$ Versión 7.0 (StatSoft, Inc., 2007) y el ajuste de la curva se realizó con el software OriginPro ${ }^{\circledR}$ Versión 8.0 (OriginLab Corporation, 2007).

\section{RESULTADOS Y DISCUSION}

EI ANOVA para propiedades texturales del mango variedad "Puerco" y "Corazón" muestra que existen diferencias significativas ( $5 \%$ de significancia) entre los diferentes EM y la variedad, para la dureza y adhesividad; sin embargo la cohesividad, gomosidad, elasticidad y masticabilidad solo presentan diferencias significativas con los EM. El test de Tukey muestra que la dureza, gomosidad, elasticidad y masticabilidad disminuyen con los EM. Sin embargo, a excepción de la dureza que presenta diferencias significativas en todos los EM, en los demas parámetros sólo se presentan diferencias significativas entre dos grupos de EM (grupo de EM 1 y 2 diferentes al grupo de EM 3, 4, 5 y 6). Cabe destacar que a partir de EM2, la transformación de pectinas en pectinas solubles es mayor, al igual que la acumulación agua y azucares. Estas transformaciones son mas leves a partir del EM3, lo que concuerda con lo reportado por Caner et al. (2008) para fresas.

EI ANOVA para propiedades texturales de la papaya variedad Hawaiana muestra que sólo existen diferencias significativas entre los diferentes estados de madurez para la dureza, adhesividad, gomosidad y masticabilidad. El sentido de la carga (transversal y perpendicular a la dirección de la fibra) no presentan diferencias significativas, por lo que se puede afirmar que la papaya es un alimento isotrópico, es decir con estructura de tejidos homogénea. El test de Tukey muestra que la dureza disminuye con el estado de madurez, sin embargo, no se presentan diferencias significativas entre los estados de madurez intermedios (EM3, EM4, EM5 y EM6), en la adhesividad, gomosidad y elasticidad, presentando el mismo comportamiento que el mango variedad "Puerco" y "Corazón".

EI ANOVA para las propiedades texturales en el plátano, muestra que existen diferencias significativas entre los diferentes estados de madurez para la dureza, adhesividad, gomosidad y masticabilidad. En la dureza se presentaron diferencias significativas en el sentido de la carga, por lo que el plátano puede ser catalogado como un alimento anisotrópico, es decir que la estructura de los tejidos no es homogénea, esto concuerda con lo reportado por Ciro et al. (2008) para plátano (Musa AAB Simmonds) y por Torres et al. (2012) para platano (Mussa Cavendish) con un análisis de relajación de esfuerzo. El test de Tukey muestra que la dureza, gomosidad y masticabilidad tienen el mismo comportamiento, presentando los EM 1, 2 y 3 una mayor magnitud en éstas propiedades que los EM 4, 5, 6 y 7, los cuales no presentan diferencias significativas entre sí. La elasticidad disminuye cuando aumenta el EM, y el EM1 presentó diferencias significativas con los demás estados de madurez, sin embargo entre los EM 2, 3, 4, 5 y 6, no se presentaron diferencias significativas.

Finalmente, los datos experimentales de parámetros de color en el espacio de color CIELAB muestran que la dureza, gomosidad y masticabilidad, presentan un buen ajuste polinómico de segundo grado en ${ }^{\circ} \mathrm{HUE}$, para la papaya variedad Hawaiana y los mangos variedad "Puerco" y "Corazón"; el plátano sólo presentó un buen ajuste para la dureza (ver Tabla 1), lo que permite inferir que la dureza es uno de los mejores parametros de madurez para las frutas estudiadas.

\section{CONCLUSIONES}

Es evidente que el perfil de textura aporta una ayuda indiscutible en la apreciación de la textura de los productos alimenticios. Los parámetros texturales del mango de "Corazón" son mayores en magnitud que en el mango de "Puerco". La dureza disminuye con el EM de las frutas, mientras que la gomosidad y masticabilidad no presentan variación entre estados de madurez intermedios.

La dureza, gomosidad y masticabilidad pueden correlacionarse con una ecuación polinómica de segundo grado en 'HUE, para el mango (Mangifera indica L) variedad "Puerco" y "Corazón"; la dureza y gomosidad para la papaya (Carica papaya L.) variedad Hawaiana y con la dureza para plátano (Mussa Cavendish).

La dureza del plátano, bajo cargas compresivas poseé más resistencia a la falla en dirección longitudinal que transversal indicando una alta presencia anisotrópica, mientras que la papaya variedad Hawaiana, no presentó diferencias significativas, indicando presencia isotrópica. 
Tabla 1. Modelos estadísticos para relación entre color y parámetros texturales para mango variedad "Puerco" y "Corazón", papaya variedad Hawaiana y el plátano

\begin{tabular}{|c|c|c|}
\hline Fruta & Ecuación & $\mathbf{R}^{2}$ \\
\hline Mango variedad "Puerco" & $\begin{array}{l}\text { Dureza }=3191,0-70,9^{\circ} \mathrm{HUE}+0,4^{\circ} \mathrm{HUE}^{2} \\
\text { Gomosidad }=2376,6 \quad 52,0^{\circ} \mathrm{HUE}+0,3^{\circ} \mathrm{HUE}^{2} \\
\text { Masticabilidad }=2989,3 \quad 65,5^{\circ} \mathrm{HUE}+0,4^{\circ} \mathrm{HUE}^{2}\end{array}$ & $\begin{array}{l}0,9678 \\
0,8389 \\
0,7838\end{array}$ \\
\hline Mango variedad "Corazón" & $\begin{array}{l}\text { Dureza }=3842,4 \quad 84,3^{\circ} \mathrm{HUE}+0,5^{\circ} \mathrm{HUE}^{2} \\
\text { Gomosidad }=6551,0 \quad 136,3^{\circ} \mathrm{HUE}+0,7^{\circ} \mathrm{HUE}^{2} \\
\text { Masticabilidad }=8664,6 \quad 179,3^{\circ} \mathrm{HUE}+0,9^{\circ} \mathrm{HUE}^{2}\end{array}$ & $\begin{array}{l}0,9078 \\
0,8139 \\
0,7881\end{array}$ \\
\hline Papaya variedad Hawaiana & $\begin{array}{l}\text { Dureza }=197,7 \quad 4,4^{\circ} H U E+0,03^{\circ} \mathrm{HUE}^{2} \\
\text { Gomosidad }=28,15 \quad 0,64^{\circ} \mathrm{HUE}+0,004^{\circ} \mathrm{HUE}^{2} \\
\text { Masticabilidad }=56,27 \quad 1,28^{\circ} \mathrm{HUE}+0,01^{\circ} \mathrm{HUE}^{2}\end{array}$ & $\begin{array}{l}0,9025 \\
0,8451 \\
0,6388\end{array}$ \\
\hline Plátano & Dureza $=181,20+348^{\circ} H U E \quad 0,02^{\circ} H_{U E}^{2}$ & 0,9376 \\
\hline
\end{tabular}

\section{REFERENCIAS}

Abdullah, M., L. Guan, K. Lim y A. Karim. The applications of computer vision system and tomographic radar imaging for assessing physical properties of food. Journal of Food Engineering, 61(1), 125-135 (2001).

Alvarez, M., W. Canet y E. López. Influence of deformation rate and degree of compression on textural parameters of potato and apple tissues in texture profile analysis. European Food Research and Technology, 215(1), 13-20 (2002).

Bourne, M. Food Texture and Viscosity: Concept and Measurement. Academic Press, San Diego, p 13-16 y 107-113 (2002).

Caner, C., M.S. Aday y M. Demir. Extending the quality of fresh strawberries by equilibrium modified atmosphere packaging. European Food Research and Technology, 227(6), 1575-1583 (2008).

Ciro, $\mathrm{H}$ y J. Osorio. Avance experimental de la ingeniería de postcosecha de frutas colombianas: resistencia mecánica para frutos de uchuva (Physalis peruviana I). Revista Dyna, 154, 39-46 (2008).

Ciro, H., S. Largo y H. Casafús. Caracterización reológica de la yuca (manihot esculenta crantz) parte i: respuesta a la compresión unidireccional. Revista Dyna, 151, 25-36 (2007).

Ciro, H., F. Monroy y E. Cortés. Estudio preliminar del comportamiento reologico bajo compresión unidireccional de la pulpa de plátano (Musa AAB Simmonds). Revista Dyna, 155, 155-161 (2008).

Ciro, H., D. Vahos y C. Márquez. Estudio experimental de la fuerza de Fractura en frutas tropicales: el tomate de Árbol (Cyphomandra betacea Sendt). Revista Dyna, 146, 55-64 (2005).

Ciro, H., D. Vahos y E. Cortes. Fuerza de fractura superficial en granadilla (Passiflora ligularis Juss) y tomate de árbol (Cyphomandra betacea Sendt): estudio experimental. Revista Facultad Nacional de Agronomía, 56(2), 2067-2076 (2003).

Correa, P., J. De la Plaza y M. Ruiz. Ensayo no destructivo para la evaluación de la madurez postrecoleción de aguacate. Agro-Ciencia, 11(2), 197-200 (1995).

Dadzie, B. y J. Orchard. Evaluación rutinaria postcosecha de híbridos de bananos y plátanos: criterios y métodos. Guías técnicas INIBAP 2. Instituto Internacional de Recursos Fitogenéticos 2, 76 (1997). 
Huang, M., J. Kennedy, B. Li, X. Xu y B. Xie. Characters of rice starch gel modified by gellan, carrageenan, and glucomannan: A texture profile analysis study. Carbohydrate Polymers, 69(3), 411-418 (2007)

Kajuna, S., W. Bilanski y G. Mittal. Textural changes of Banana and Plantain pulp during ripening. Journal of the Science of Food and Agriculture, 75(2), 244-250 (1997).

Mendoza, F., P. Dejmek y J. Aguilera. Calibrated color measurements of agricultural foods using image analysis. Postharvest Biology and Technology, 41(3), 285-295 (2006).

Ospina, D., H. Ciro y I. Aristizábal. Determinación de la fuerza de la fractura superficial y fuerza de firmeza en frutas de lulo (Solanum quitoense $x$ Solanum hirtum). Revista Facultad Nacional de Agronomía, 60(2), 4163-4178 (2007).

OriginLab Corporation. OriginPro 8 SR0 versión 8.072. www.OriginLab.com. (2007).

Pedreschi, F., D. Mery, F. Mendoza y J. Aguilera. Classification of potato chips using pattern recognition. Journal of food science, 69 (6), 264-269 (2004).

Peña, M. Tratamiento cuarentenario contra la mosca mediterráneo (Ceratitiscapitata) en mangos variedad Haden (Manguífera índica) con irradiación gamma (Co-60). Magíster en Ciencia de los Alimentos, Universidad Nacional Mayor de San Marcos, Lima (2008).

Santamaría, F., E. Sauri, F. Espadas, R. Díaz, A. Larqué y J. Santamaría. Postharvest ripening and maturity indices for maradol papaya. Interciencia, 34(8), 583- 588 (2009).

Segnini, S., P. Dejmek y R. Öste. A low cost video technique for color measurement of potato chips. LWT Food Science and Technology, 32(4), 216-222 (1999).

Sharma, G., W. Wencheng y E. Dalal. The CIEDE2000 Color-Difference Formula: Implementation notes, Supplementary test data, and mathematical observations. COLOR research and application, 30 (1), 21-30 2005.

StatSoft, Inc. STATISTICA (data analysis software system), versión 7. www.statsoft.com_2007).

Torres, R., E. Montes, O. Pérez y R.D. Andrade, Influencia del estado de madurez sobre las propiedades Viscoelásticas de frutas tropicales (mango, papaya y plátano). Información Tecnológica, 23(5),115-124 (2012).

Zuñiga, L., H. Ciro y J. Osorio. Estudio de la dureza del queso Edam por medio de análisis de perfil de textura y penetrometría por esfera. Revista Facultad Nacional de Agronomía, 60 (1), 3797-3811 (2007). 
